\title{
VESTIGIOS COLONIALES EN LA REPRESENTACIÓN DEL OTRO: UN RETO PARA LA COMUNICACIÓN DECOLONIZADORA
}

\author{
Eduardo César Augusto Muro Ampuero* \\ Universidad de Bonn, Alemania \\ eduardomuroa@gmail.com
}

Recibido: 26/10/2019/ Aceptado: 10/2/2020

doi: $10.26439 /$ contratexto2020.n033.4788

Resumen. El artículo tiene como objetivo principal presentar la importancia y relevancia de los diversos medios de representación en la construcción de la imagen del Otro (para ser más específicos, el Otro colonizado). Se considera, además, que este tipo de representación de la alteridad viene acompañada de una cierta teoría de la verdad, la cual establece jerarquías respecto a quiénes pueden pertenecer al grupo, qué tipo de conocimiento es válido, qué formas de espiritualidad son aceptadas, entre otras diferenciaciones entre "Nosotros" y "Ellos". En ese sentido, en este trabajo se intenta esbozar, por un lado, cómo a partir de una cierta forma de producción de conocimiento se origina un paradigma en donde las representaciones de lo indígena ocupan un lugar subordinado en las relaciones intersubjetivas de nuestras sociedades (siendo estas materializadas en el cuerpo de este Otro). Por otro lado, se mencionan algunos ejemplos de estos vestigios representacionales de lo indígena, todavía presentes en la sociedad peruana, sobre todo difundidos a través de los medios de comunicación. Y, por último, se reflexiona sobre los dos primeros puntos en el marco de una comunicación decolonizadora.

Palabras clave: medios de representación / comunicación / decolonización / otredad / indigeneidad

\footnotetext{
* Estudiante de la Maestría en Estudios Culturales de Latinoamérica en la Universidad de Bonn. Magíster en Estudios del Discurso por la Universitat Pompeu Fabra. Bachiller en Comunicación por la Universidad de Lima (https://orcid.org/0000-0002-7052-302).
} 


\section{COLONIAL VESTIGES IN THE REPRESENTATION OF THE OTHER: A CHALLENGE FOR A DECOLONIZING COMMUNICATION}

Abstract. The main objective of this article is to show the importance and relevance of various means of representation in the construction of the image of the Other (more specifically, the colonized Other). It is also considered that this type of representation of otherness is accompanied by a certain theory of truth, which establishes hierarchies regarding who can belong to the group, what type of knowledge is valid, what forms of spirituality are accepted, among other differences between "We" and "They". In that sense, we aim to outline in this research, on the one hand, how a paradigm is produced from a certain form of knowledge production, where the representations of the indigenous occupy a subordinate place in the intersubjective relations of our societies (also materialized in the body of this Other). On the other hand, we mention some examples of these representational vestiges of the indigenous, which are still present in the Peruvian society and mainly disseminated through the media. Finally, we reflect on the first two topics concerning the decolonizing communication.

Keywords: means of representation / communication / decolonizing / otherness / indigeneity

\section{VESTÍGIOS COLONIAIS NA REPRESENTAÇ̃̃O DO OUTRO: UM DESAFIO PARA A COMUNICAÇÃOO DECOLONIZADORA}

Resumo. 0 principal objetivo deste artigo é apresentar a importância e a relevância dos vários meios de representação na construção da imagem do Outro (mais especificamente, do Outro colonizado). Considera-se também que este tipo de representação da alteridade é acompanhado por uma certa teoria da verdade, que estabelece hierarquias sobre quem pode pertencer ao grupo, que tipo de conhecimento é válido, que formas de espiritualidade são aceites, entre outras diferenciações entre "Nós" e "Eles". Nesse sentido, neste trabalho tentamos delinear, por um lado, como a partir de uma certa forma de produção de conhecimento produz-se um paradigma onde as representações dos indígenas ocupam um lugar subordinado nas relações intersubjetivas das nossas sociedades (sendo estas materializadas no corpo deste Outro). Por outro lado, são citados alguns exemplos desses vestígios representacionais dos indígenas, ainda presentes na sociedade peruana, sobretudo, disseminados pela mídia. E, finalmente, reflectimos sobre os dois primeiros pontos no quadro de uma comunicação descolonizante.

Palavras-chave: meios de representação / comunicação / decolonização / alteridade / indigeneidade 


\section{INTRODUCCIÓN}

Cuando Deloria afirma que un paso fundamental para "ejercer los derechos soberanos de las tribus es a través del control de los medios de representación" (citado por Lonetree, 2012, p. 81), a nuestro parecer, sitúa no solo la problemática de cómo las comunidades indígenas son retratadas en los diversos espacios donde esta representación se pueda dar (en un nivel discursivo que puede expresarse en diversos espacios como, por ejemplo, los museos, las instituciones públicas o los medios de comunicación), sino también, en términos generales, la importancia de la representación de la Otredad (a través de diversos canales) en el imaginario colectivo. Sin embargo, si tenemos en cuenta quiénes son los que controlan estos medios y el hecho de que existe una distribución asimétrica de representación de voces "otras"1 (por motivos históricos, económicos, sociales y culturales), la toma de estos medios constituye una de las tareas más difíciles a las que nos podemos enfrentar en la actualidad ${ }^{2}$. No solo por los intereses económicos que están en juego, sino también por la fuerte impronta de una ideología capitalista y "occidentalista" 3 del mundo, en la cual se genera una relación asimétrica, con una impronta jerárquica epistémica (entre conocimientos y saberes), como también socioeconómica, entre los países que conforman el "Occidente desarrollado" y los demás.

Así, partiendo del escueto contexto descrito en el párrafo anterior, podemos considerar la relevancia de los medios de representación como espacios (y herramientas) para que las comunidades indígenas (o la Otredad, en términos más amplios) puedan expresarse y representarse en sus propios términos.

No obstante, la gran asimetría hoy existente circunscribe a las comunidades indígenas a un plano marginal, de resistencia. $Y$ ello es algo que se viene arrastrando desde

1 Sobre el uso del pronombre otras/otros (y sus variantes) después del sustantivo, remitimos a lo dicho por la profesora Catherine Walsh (2009): “La noción de 'otro/otra' aquí, como he explicado anteriormente, no implica una sociedad o un proyecto más; tampoco un proyecto alternativo que tenga como referente al actual. Más bien parte del sentido que le da el árabe-islámico Abdelkebir Khatibi (2001), retomado por el argentino-estadounidense Walter Mignolo (2003). Es decir, al encontrar y construir algo radicalmente distinto desde la diferencia y hacia la liberación (Khatibi); la necesidad de imaginar un proyecto de sociedad no desde la modernidad o los colonizadores y "liberadores", sino desde principios políticos, éticos, económicos y epistémicos distintos (Mignolo, 2003)" (p. 40).

2 Si bien aquí aludimos especialmente a los medios "tradicionales" (radio, televisión, prensa escrita), consideramos que estas dificultades también se presentan en los medios (y/o plataformas) digitales. Teniendo en cuenta los roles que desempeñan estos últimos medios en las comunidades indígenas (y también afros) como espacios importantes de autorrepresentación y expresión de sus identidades (porque los expone a un público mayor, a nivel global), afirmamos que, dentro de la ecología mediática, estas son prácticas que no llegan a competir cuantitativamente con las industrias culturales "occidentales", capitalistas, machistas y eurocéntricas.

3 De aquí en adelante, cada vez que hagamos referencia a "Occidente" (y sus derivados), lo haremos entre comillas con la finalidad de especificar, teniendo en cuenta lo dicho por Said (2016), que la ubicación de "Occidente" es relativa al locus de enunciación y que el "Occidente" que mencionamos es a lo que comúnmente se ha denominado como cultura occidental. 
la Colonia, negando, por lo tanto, cualquier intento de diálogo, en el sentido bajtiniano 4 entre las diversas expresiones y representaciones culturales (ya que unas se imponen sobre las otras). Por tanto, los objetivos de este trabajo son (i) mostrar cómo a partir de una cierta forma de producción de conocimiento (con directas implicaciones en la historia como disciplina) se produce un paradigma en donde las representaciones de lo indígena ocupan un lugar subordinado en las relaciones intersubjetivas de las sociedades; (ii) mencionar algunos ejemplos sobre estos vestigios representacionales de lo indígena que aún están presentes en la sociedad peruana, sobre todo, difundidos a través de los medios de comunicación (si los consideramos como fuentes contemporáneas y sincrónicas para registrar eventos históricos); y (iii) reflexionar sobre lo anterior en el marco de una comunicación decolonizadora. En cada uno de los puntos mencionados se realiza un breve análisis exploratorio de algunas teorías y conceptos que nos aproximen al tema y nos ayuden a comprender el punto en cuestión.

\section{LA COLONIALIDAD EN EL OTRO COLONIZADO}

Desde la Antigüedad, ha existido en la tradición filosófica una distinción entre episteme y doxa, es decir, entre un saber determinado y un saber indeterminado, entre conocimiento/ciencia y saber (Manrique Tisnés, 2008, p. 99). Esta distinción ha permeado el debate intelectual contemporáneo con respecto a dónde situar y cómo nombrar a los saberes indígenas frente al conocimiento producido por las disciplinas científicas, teniendo en cuenta "las condiciones actuales de asimetría, dominación y subalternidad" (Pérez y Argueta, 2011, p. 48) ${ }^{5}$.

4 El dialogismo de Bajtín es una teoría discursiva que se constituye también como un posicionamiento ético, ya que considera que el diálogo es un intercambio intersubjetivo "entre dos sujetos radicalmente distintos, pero con valor propio y autónomo equivalente" (Bubnova, 1997, p. 260) y que tienen la responsabilidad de escucharse y responderse (Nealon, 1997, p. 131). De esa manera, dialogar implica el reconocimiento y aceptación a priori del Otro, pues al considerar a la persona como una "compleja estructura dialógica" (Bubnova, 1997, p. 260) sitúa su fundación ontológica no en el "yo soy", sino que en el "yo también", lo cual implica la existencia sine qua non de un "tú eres" (Bubnova, 1997, p. 264). Solo a través del reconocimiento y de la aceptación de la autonomía del yo y del Otro, así como de la radical distinción entre ambas, es que se puede dar un diálogo (de escucha y respuesta) honesto y responsable.

5 A diferencia de la clásica distinción entre episteme y doxa, de la entrevista con la activista y socióloga boliviana Silvia Rivera Cusicanqui (2016) en la revista Ojarasca se puede inferir que para ella episteme es un paradigma, una forma de enfrentarse al mundo y de relacionarse con él, es decir, es la relación que los sujetos construyen (subjetiva e intersubjetivamente) a lo largo de su devenir histórico en comunidad (e intercomunalmente) y en estrecha relación con el espacio que les rodea. Son racionalidades que buscan darle sentido al mundo en el que viven. Es decir que para ella episteme sería, en contraste con la idea única de que esta es la producción de conocimiento científico, el modelo por medio del cual los sujetos (re)producen sus formas contingentes de ser y de estar en el mundo. 
El desarrollo de muchos de estos saberes no solo se ha visto socavado por el carácter expansivo y colonizador de sociedades que ostentaban un poder mucho mayor (ya sea militar, económico o político), sino también porque iba acompañado de una cierta teoría de la verdad (Dussel, 2007, p. 293). Esta combinación coloca en una posición privilegiada a las formas de conocer el mundo de unos respecto de la de otros (estando estos últimos equivocados o en un estadio previo de aprehensión del mundo), lo que generó relaciones asimétricas entre aquellos que poseían el poder y aquellos que no y, en el caso de las colonias, entre colonizadores y colonizados.

El descubrimiento de las Américas para Europa, es decir, la "aparición" del continente americano en el panorama europeo, tuvo diversas connotaciones desde los primeros contactos que se establecieron entre los unos y los otros. Para Quijano (2000), uno de los principales núcleos de la clasificación social que se fue gestando durante esas épocas se dio a través de la idea de raza. Estas distinciones, basadas en "supuestas estructuras biológicas diferenciales entre estos grupos" (p. 202), tuvieron como consecuencia que "los pueblos conquistados y dominados fuer[a]n situados en una posición natural de inferioridad, [...] así como [también] sus descubrimientos mentales y culturales" (p. 203). En otras palabras, una de las racionalizaciones de estas diferencias (de la colonialidad del poder) se llevó a cabo a través de concepciones esencialistas fundadas en una supuesta relación interdependiente entre raza y cultura. De esa manera, Europa "concentró bajo su hegemonía el control de todas las formas de control de la subjetividad de la cultura, y en especial del conocimiento, de la producción de conocimiento" (Quijano, 2000, p. 209).

Además de esta colonialidad del poder, que también toma en cuenta las relaciones asimétricas sustentadas en el control del trabajo y la emergencia del capitalismo, Walsh (2007) menciona otras tres esferas que forman parte de la colonialidad: la colonialidad del saber, que sitúa al eurocentrismo como única forma de conocimiento y que, por consiguiente, implica el descarte de conocimientos "otros" (como el de los indígenas o afros) (p. 104); la colonialidad del ser, que implica la imposición, control y persecución de unos seres sobre otros (p. 105); y la colonialidad de la naturaleza, que es la "división binaria cartesiana entre naturaleza y sociedad, [...] que descarta por completo la relación milenaria entre seres, plantas y animales, como también entre ellos, los mundos espirituales y los ancestros" (p. 106).

Si bien todas estas esferas son interdependientes (cuyas distinciones son analíticas), nos gustaría hacer énfasis en la esfera del saber, de la producción de conocimiento. Esta colonialidad, como dice Walsh (2007), “[...] no solo estableció el eurocentrismo como perspectiva única de conocimiento, sino que al mismo tiempo descartó por completo la producción intelectual indígena y afro como 'conocimiento' y, consecuentemente, su capacidad intelectual" (p. 104). Ello se puede apreciar, por ejemplo, en la concepción que muchos pensadores, antes y después de la conquista, tenían de la historia, uno 
de cuyos aspectos principales partía de una teoría de la escritura que situaba a esta como el mejor invento de la humanidad, capaz de domar la voz (Mignolo, 1995, p. 294). Como consecuencia directa de esta teoría, en la historiografía "occidental" se consideró que aquellas culturas que no habían conseguido domar la voz a través de las palabras carecían de la precisión para hacer historia, inclusive su propia historia, como dijo Juan de Torquemada, misionero e historiador franciscano, respecto a la pictografía azteca (Mignolo, 1994, p. 300).

El concepto de historia (o historiografía) de Torquemada implica la dominante definición ciceroniana de la misma, la cual se forjó a partir de la experiencia de las narraciones escritas alfabéticas realizadas por los historiadores griegos y romanos, así como del legado retórico de la Roma imperial (principalmente ciceroniana y quintiliana). Fue la creencia en la conservación exacta de la memoria y la glorificación del pasado mediante la escritura alfabética lo que dio lugar a una poderosa complicidad entre el poder de la letra y la autoridad de la historia. (Mignolo, 1995, pp. 128-129)6

Sobre esta complicidad Mignolo (1995) continúa:

La escritura de cartas como medio de comunicación era una práctica común hacia finales del siglo Xv entre los humanistas. Durante el siglo Xvı también se convirtió en un instrumento fundamental de control administrativo y de gobierno. La escritura alfabética y la escritura de cartas (epistola) como género tuvo en su momento una larga historia en Occidente y durante el proceso de colonización generó numerosas situaciones comunicativas que fueron narradas en cientos de anécdotas que van desde el Perú colonial hasta la Nouvelle France (Canadá). (p. 172)

En ese sentido, nos parece interesante mencionar la relación que hace Glissant entre literatura e historia como herramientas para subordinar otras formas de registrar eventos pasados y controlar la memoria:

La historia es una fantasía altamente funcional de Occidente, originada precisamente en el momento en que "hizo" sola la historia del Mundo [...]. En esta etapa, la Historia se escribe con $\mathrm{H}$ mayúscula. Es una totalidad que excluye otras historias

6 "Torquemada's concept of history (or historiography) implies the dominant Ciceronian definition of it, which was forged on the experience of alphabetic written narratives accomplished by Greek and Roman historians, as well as the rhetorical legacy of imperial Rome (mainly Ciceronian and Quintilian). It was the belief in the accurate preservation of memory and the glorification of the past by means of alphabetic writing that resulted in a powerful complicity between the power of the letter and the authority of history" (Mignolo, 1995, pp. 128-129).

7 "Letter writing as a mean of communication was a common practice toward the end of the fifteenth century among the humanists. During the sixteenth century it also became a fundamental instrument of administrative control and government. Alphabetic writing and letter writing (epistola) as a genre had at the time a long history in the West and during the process of colonization generated numerous communicative situations that were narrated in hundreds of anecdotes ranging from colonial Peru to colonial Nouvelle France (Canada)" (Mignolo, 1995, p. 172). 
que no encajan en la de Occidente [...]. La literatura alcanza una metaexistencia, la omnipotencia de un signo sagrado, que permitirá a los pueblos con escritura pensar que está justificado dominar y gobernar a los pueblos con una civilización oral [...]. Es de nuevo esta doble hegemonía de una Historia con $\mathrm{H}$ mayúscula y de una Literatura consagrada por el poder absoluto del signo escrito contra la que los pueblos que hasta ahora habitaban el lado oculto de la tierra lucharon, al mismo tiempo que luchaban por el alimento y la libertad. (Citado por Mignolo, 1995, p. 127)

Esta impronta epistemológica en la historiografía de "Occidente", es decir, la hegemonía de la Historia con $\mathrm{H}$ mayúscula, implicó que todos aquellos eventos pasados que no fueron registrados a través de la escritura no fuesen considerados como fuentes precisas (o, inclusive, como tales). En las Américas, según Salomon (1999), esta distinción separaría a una "historia sobre los indios" (Indian histories) de una "historia de los indios" (history of Indians). La diferencia estribaría en que la primera es heredera de la historiografía "occidental", mientras que la segunda se aproximaría a la historia desde la perspectiva de los propios indígenas. De esa manera, se tienen en cuenta otros métodos de rememoración (Salomon, 1999, p. 20), como los quipus, los textiles, la tradición oral, las cerámicas y también, como señala Santos Granero $(1998,2004)$, la escritura topográfica, entre otros.

Asimismo, esta hegemonía de la Historia, a través de la escritura o literacidad, ha sido usada para determinar las fronteras entre el pasado y el presente (Tuhiwai, 2008, p. 28). Esta división se encuentra también en otras disciplinas de las ciencias sociales, como bien señala Fabian (2014), bajo una distinción temporal entre los que estudian y los que son estudiados, es decir, entre el investigador y el objeto de estudio. Esta diferenciación radica en una temporalización evolucionaria de la historia de la humanidad con "Occidente" a la cabeza.

8 "History is a highly functional fantasy of the West, originating at precisely the time when it alone "made" the history of the World [...]. At this stage, History is written with a capital H. It is a totality that excludes other histories that do not fit into that of the West [...]. Literature attains a metaexistence, the all-powerfulness of a sacred sign, which will allow people with writing to think it justified to dominate and rule peoples with an oral civilization [...]. It is again this double hegemony of a History with a capital $\mathrm{H}$ and a Literature consecrated by the absolute power of the written sign that the peoples who until now inhabited the hidden side of the earth fought, at the same time they were fighting for food and freedom" (Glissant, citado por Mignolo, 1995, p. 127).

9 Sobre la relación entre la investigación y el imperialismo y el colonialismo europeo, Tuhiwai (2008) dice lo siguiente: "Esta memoria colectiva del imperialismo se ha perpetuado a través de las formas en que el conocimiento sobre los pueblos indígenas fue recogido, clasificado y luego representado de diversas maneras de vuelta a Occidente, y luego, a través de los ojos de Occidente, de vuelta a los que han sido colonizados [This collective memory of imperialism has been perpetuated through the ways in which knowledge about indigenous peoples was collected, classified and then represented in various ways back to the West, and then, through the eyes of the West, back to those who have been colonized]" (p. 1). 
La antropología surgió y se estableció como un discurso alocrónico; es una ciencia de otros hombres en otro tiempo. Es un discurso cuyo referente ha sido eliminado del presente del sujeto que habla/escribe. Como las relaciones entre los pueblos y las sociedades que estudian y las que son estudiadas, las relaciones entre la antropología y su objeto son inevitablemente políticas [...]. Entre las condiciones históricas en las que surgió nuestra disciplina y que afectaron a su crecimiento y diferenciación estaban el surgimiento del capitalismo y su expansión colonialista-imperialista en las mismas sociedades que se convirtieron en el objetivo de nuestras investigaciones. Para que esto ocurriera, las sociedades expansivas, agresivas y opresivas que colectiva e inexactamente llamamos Occidente necesitaban espacio para ocupar. Más profunda y problemáticamente, necesitaban Tiempo para acomodar los esquemas de una historia unidireccional: progreso, desarrollo, modernidad (y un espejo con los negativos de las imágenes: estancamiento, subdesarrollo, tradición). En resumen, la geopolítica tiene sus fundamentos ideológicos en la cronopolítica. (Fabian, 2014, pp. 143-144) ${ }^{10}$

En ese sentido, podemos decir que la Otredad ha tenido un lugar subordinado, cuya representación ha sido realizada por aquellos que estaban en el poder, por aquellos que han tenido los medios y los espacios para moldear la Otredad a través de sus propios ojos, pero no a través de los ojos de la Otredad misma"1.

De esa manera, los paradigmas "otros", que son totalmente diferentes del paradigma de "Occidente", se encuentran no solo en una posición marginal, sino también en un pasado del cual pueden escapar únicamente por medio del patronazgo "occidental". Esto, a grandes rasgos, implicaría el abandono de sus saberes, de sus formas de estar en el mundo y de enfrentarse a él en nombre, por ejemplo, de la modernidad. La tarea

10 "Anthropology emerged and established itself as an allochronic discourse; it is a science of other men in another Time. It is a discourse whose referent has been removed from the present of the speaking/writing subject. [...] As relationships between peoples and societies that study and those that are studied, relationships between anthropology and its object are inevitably political [...] Among the historical conditions under which our discipline emerged and which affected its growth and differentiation were the rise of capitalism and its colonialist-imperialist expansion into the very societies which became the target of our inquiries. For this to occur, the expansive, aggressive, and oppressive societies which we collectively and inaccurately call the West needed Space to occupy. More profoundly and problematically, they required Time to accommodate the schemes of a one-way history: progress, development, modernity (and their negative mirror images: stagnation, underdevelopment, tradition). In short, geopolitics has its ideological foundations in chronopolitics" (Fabian, 2014, pp. 143-144).

11 Con relación a esta subordinación e invisibilización del Otro colonizado por parte de aquellos que están en el poder, De Sousa Santos (2012) dice lo siguiente respecto a la carga bastante eurocéntrica de los proyectos independentistas del siglo XIX: "Entre los vínculos eurocéntricos que condicionaron las independencias del siglo XIX se encuentran el Estado y el derecho, concebidos como monolíticos y monoculturales, el capitalismo dependiente, el colonialismo interno, el racismo, el autoritarismo y el centralismo burocráticos, y el canon cultural occidental. Sobre esta base se diseñaron políticas económicas, educativas, culturales, lingüísticas, sanitarias, de seguridad, asistencialistas, territoriales fundadas en la exclusión, represión o invisibilización de las maneras de vivir, pensar, actuar y sentir en colisión con los principios nacionalistas liberales" (p. 14). 
recaería en decolonizar las cuatro esferas mencionadas por Walsh: el poder, el saber, el ser y la naturaleza.

\section{VESTIGIOS COLONIALES: NUEVOS RETOS, VIEJOS PROBLEMAS}

En el Perú, es pan de cada día escuchar a los amigos, a la familia, a conocidos, etcétera, decir algún comentario racista, discriminador o clasista. Hacer bromas con base en estereotipos burdos sobre personas afrodescendientes o andinas, reírse del acento de una persona que vive en la selva o en la sierra y cuya lengua materna no sea el castellano. Tener expectativas de que algún miembro de la familia se case con una persona europea, o por lo menos blanca, para "mejorar la raza", como una forma de ascender en las jerarquías sociales. 0 escuchar a alguien decir "ni que fueras europeo" (más específicamente, de algún país de Europa del Norte), cuando presencia un comentario racista explícito de una persona que no es blanca. Uno nunca quiere ser el cholo del otro, sino que busca la manera de blanquearse frente al otro para poder diferenciarse de él y estar por encima de él. Lo indígena (pero también lo afro) es representado a través de estos microrracismos, parte constituyente de las microfísicas del poder que estructuran a la sociedad peruana. Pero, claro, cuando alguien es interpelado sobre dichos comentarios, ese alguien no estaba siendo racista o discriminador e intenta matizar sus palabras, o justificarse, mediante una "objetividad" acrítica (afirmando que el mundo es así). Sin embargo, como hemos visto líneas atrás, este no es un problema de la actualidad, sino que se ha heredado de la Colonia, y se nutre y perpetúa a través de la colonialidad.

No obstante, esto no es algo que se haya quedado en el plano de las interacciones cotidianas, sino que estos mensajes también encuentran eco en los diversos medios de comunicación: en programas humorísticos (La paisana Jacinta ${ }^{12}$, por ejemplo), reportajes periodísticos, comentarios de personajes públicos, tuits, videos de YouTube, páginas de

12 La paisana Jacinta era un programa humorístico televisivo que narraba las peripecias de una mujer andina y de escasos recursos económicos en la capital peruana, como una especie de choque de dos mundos: el andino y el capitalino, el rural y el urbano, lo moderno y lo atrasado, por poner algunos ejemplos. Cargado de estereotipos negativos sobre la imagen de la protagonista, Jacinta (en representación de la mujer andina) es retratada como sucia (ya que, como se puede apreciar en diversos capítulos, huele, o apesta, a llama/alpaca/vicuña), sin algunos dientes (porque según este imaginario la mujer andina es así), ignorante y fácil de engañar por su escasa o nula educación (aunque por esos azares de la narrativa audiovisual ella termina siempre triunfante en los problemas que se le presenta en cada sketch, como una especie de castigo divino a aquellos que se querían aprovechar de ella), entre otras características. Si bien al final de cada capítulo Jacinta sale airosa por medio de su "inocente viveza", ello no quita que durante todo el episodio se haya hecho uso de las características ya mencionadas para causar gracia. La paisana Jacinta estuvo al aire hasta el 2015, luego formó parte de otro programa humorístico ( $E l$ wasap de JB) y a fines del 2019 fue retirado del aire por orden del Juzgado Civil de Wánchaq (Cusco, Perú), que aceptó la demanda de amparo de lideresas campesinas cusqueñas contra la representación del personaje de Jacinta. 
Facebook, Instagram, etcétera ${ }^{13}$. Es decir que el nivel de penetración de estos mensajes se ha ampliado exponencialmente ${ }^{14}$.

Como lo expone Mignolo (1995) en los casos de Pedro Mártir de Anglería (Pietro Martire d'Anghiera) y Bernardino de Sahagún, y Bauer (2001) en el de Felipe Guamán Poma de Ayala, cuando se analizan las fuentes históricas es importante tener en cuenta los códigos y los canales que han sido utilizados para registrar eventos importantes de la historia. En otras palabras, es fundamental saber quién está escribiendo (biografía del autor), sobre qué está escribiendo (es decir, qué sucesos está narrando), a quién está dirigido, cómo y dónde escribe, a saber, estilos, códigos y medios, y, por último, para qué registra lo que está escribiendo. De esa misma manera, cuando nos aproximamos a las diversas representaciones en los diferentes medios y espacios, es clave hacer todas esas preguntas: el quién, qué, a quién, de qué manera, dónde y por qué.

Pero, además, es necesario considerar, siguiendo lo dicho por Mignolo (1995), el lugar privilegiado que tuvieron (y que tienen) la escritura y la literacidad como justificaciones teóricas en la historiografía, pero también en otras ramas de la producción de conocimiento, cuyas implicaciones fueron expuestas a través de la negación de saberes "otros", de epistemes "otras". En consecuencia, la escritura y la literacidad, por medio de canales y códigos específicos para expresarse, registrar la historia y producir conocimiento, legitimaron la hegemonía de "Occidente" en el campo del saber, lo colonizaron. Pero no solo eso, sino que también generaron y diseminaron una imagen de lo indígena como alguien primitivo, carente de intelecto (Walsh, 2007, p. 105). Y es en ese sentido en el que encontramos importante el uso de los medios de representación. El que tiene el poder, el que ostenta el control de los medios de representación, tiene la potestad de representar al

13 Sobre el racismo y la colonialidad en los medios de comunicación existe una amplia bibliografía. Por ejemplo, sobre la extensión del colonialismo en el plano tecnopolítico (Elizalde, 2019); sobre reproducción y perpetuación de estereotipos de la comunidad afrodescendiente en América Latina y el Caribe (Pineda, 2016); sobre discursos racistas en las redes sociales (Arriaga, 2013; Ferrándiz, Ibáñez y Espinosa, 2011); sobre la representación de la indigeneidad en medios colombianos (González y Arteaga, 2005); sobre la mujer andina en la televisión peruana o La Paisana Jacinta (Alarcón y Torres, 2015; Dettleff, 2015; respectivamente).

14 A modo de ejemplo, lo que menciona Elizalde (2019) sobre el "colonialismo 2.0" y la concentración comunicativa y cultural en el contexto global puede arrojar luz sobre cómo unos discursos, en detrimento de otros, pueden penetrar en el imaginario colectivo: “La nueva e intensa concentración comunicativa y cultural es mucho más global que la de las industrias culturales transnacionales o nacionales que conocíamos. Una sola empresa privada de Estados Unidos, por ejemplo, decide cómo gasta un cuarto de la población mundial cerca de 50 millones de horas diarias. Su valor diferencial es que crecen los usuarios a ritmos vertiginosos con tasas gigantescas, no solo en números brutos, sino en densidad y alcance" (p. 103). A lo que se le sumaría que "[n]uestra región [América Latina] todavía tiene una alta dependencia del tráfico internacional de internet, principalmente proveniente de los Estados Unidos de Norteamérica, no solamente porque es el principal mercado de proveedores de contenidos, pero también porque muchos de los contenidos generados en la región se alojan en ese país" (CEPAL, CETiC.br y NiC.br, 2018, p. 21). 
otro de la forma como ve a este en su exterioridad. Por lo tanto, cuando hablamos de tomar los medios de representación, estamos hablando de una de las aristas de la lucha por la emancipación en todas sus dimensiones (económica, política, social y cultural).

Hay dos ejemplos que nos gustaría mencionar para enfatizar el carácter político, desde una posición privilegiada del poder, de los medios de representación. Por un lado, durante el reinado de los Reyes Católicos se realizó un proyecto humanista que buscaba en esa parte de la península ibérica "la creación de un nuevo territorio cultural" (lannuzzi, 2008, p. 56) a través de "la relevancia de las nuevas técnicas mediáticas y su utilidad en la construcción de un nuevo lenguaje religioso y político capaz de educar, unificar y disciplinar a buenos 'fieles-súbditos'" (lannuzzi, 2008, p. 38). Al respecto, el gran humanista español Antonio de Nebrija, autor de la primera gramática castellana (1492) y parte de este proyecto unificador, dijo lo siguiente sobre la utilidad de su obra en el contexto sociopolítico de su época:

[...] cuando en Salamanca di la muestra de aquesta obra a vuestra real Majestad e me preguntó que para qué podía aprovechar, el mui reverendo padre Obispo de Ávila me arrebató la respuesta; y respondiendo por mí, dixo que después que Vuestra Alteça metiesse debaxo de su iugo muchos pueblos bárbaros e naciones de peregrinas lenguas, y con el vencimiento aquellos ternían necesidad de recebir las leies quel vencedor pone al vencido, y con ella nuestra lengua, entonces por esta mi Arte podrían venir en el conocimiento della, como agora nosotros deprendemos el arte de la gramática latina para deprender el latín. I certo así es que no sola mente los enemigos de nuestra fe, que tienen ia necesidad de saber el lenguaje castellano, mas los vizcainos, navarros, franceses, italianos, y todos los otros que tienen algún trato y conversación en España y necesidad de nuestra lengua, si no vienen desde niños a la deprender por uso, podrán la más aína saber por esta mi obra [...]. (Citado por lannuzzi, 2008, pp. 49-50)

Por otro lado, y similar en contenido a lo expuesto hace más de quinientos años, el 5 de octubre del 2011 el Comité de Relaciones Exteriores del Senado de Estados Unidos presentó un reporte titulado Los gobiernos latinoamericanos necesitan ser "amigables" con las redes sociales y la tecnología15, en donde se enfatizaba la relevancia de los "nuevos medios sociales" (i. e., redes sociales) para los intereses estadounidenses:

Con más del $50 \%$ de la población del mundo menor de 30 años de edad, los nuevos medios sociales y las tecnologías asociadas, que son tan populares dentro de este grupo demográfico, seguirán revolucionando las comunicaciones en el futuro [...]. Los medios sociales y los incentivos tecnológicos en América Latina sobre la base de las realidades políticas, económicas y sociales serán

15 Título original: Latin American Governments need to "friend" social media and technology. El documento puede ser encontrado en https://www.govinfo.gov/content/pkg/CPRT-112SPRT70501/html/ CPRT-112SPRT70501.htm (revisado el 21 de febrero del 2020). 
cruciales para el éxito de los esfuerzos gubernamentales de EE. UU. en la región. (Citado por Elizalde, 2019, p. 106)

Lo que se ha querido mostrar con estos ejemplos es la función estratégica de los medios de comunicación en el plano sociopolítico. Y que, al igual que antes se usó la fuerza de la letra para unificar al reino de Castilla y para generar una distinción entre las culturas que tenían historia y las que no, con base en su literacidad y su capacidad de registrar la historia a través de la escritura, hoy se utilizan también los medios de comunicación como herramientas de la geopolítica neocolonial entre países "desarrollados" y "subdesarrollados".

De igual manera que es posible ver estos nexos, en el plano latinoamericano también es posible ver en la actualidad una diversidad de discursos que cimentan representaciones de lo indígena que vienen de antaño, a saber, lugares comunes que son vestigios de nuestra época colonial. A modo de ejemplo, nos gustaría presentar algunos casos de este tipo de narrativa.

En 1990, Mario Vargas Llosa escribió un artículo titulado Cuestiones de la conquista (Questions of Conquest, en el original ${ }^{16}$ ) en una revista estadounidense en donde argumentaba en favor de la conquista y la sociedad emergente que se gestaba en el Perú. Sin embargo, esta nueva sociedad (como otras que también emergían en la región) no se ha podido constituir como una nación real porque todavía persiste un problema básico: la difícil coexistencia de dos culturas irreconciliables ${ }^{17}$. "Dos culturas, una occidental y otra moderna, la otra aborigen y arcaica, apenas coexisten, separadas entre sí por la explotación y la discriminación que la primera ejerce sobre la segunda" (Vargas Llosa, 1990, p. 51 , las cursivas son nuestras) ${ }^{18}$.

Pero, entonces, ¿cuál sería el camino para poder constituirnos como una "nación real"? Vargas Llosa (1990) es bastante claro al respecto:

El precio que ellos [los indígenas] deben pagar por la integración es alto: la renuncia a su cultura, su idioma, sus creencias, sus tradiciones y costumbres, y la adopción de la cultura de sus antiguos maestros [...]. Si me viera obligado a elegir entre la preservación de las culturas indígenas y su completa asimilación, con gran tristeza elegiría la modernización de la población indígena, porque hay

16 La versión en línea se puede encontrar en el siguiente enlace: https://harpers.org/archive/1990/12/ questions-of-conquest/ (revisado el 15 de marzo del 2019).

17 Si bien los trabajos revisados que abordan este texto de Vargas Llosa no lo hacen desde una perspectiva comunicacional, sí examinan la estrecha relación de sus palabras con concepciones modernistas y neoliberales que se basan en una aproximación lineal de la historia, cuyo horizonte es el progreso; de esa manera, generan una distinción entre "civilizados" y "bárbaros" (Kokotovic, 2000; De Castro, 2010).

18 "Two cultures, one Western and modern, the other aboriginal and archaic, hardly coexist, separated from each other because of the exploitation and discrimination that the former exercises over the latter" (Vargas Llosa, 1990, p. 51). 
prioridades; y la primera prioridad es, por supuesto, luchar contra el hambre y la miseria. (pp. 51-52) ${ }^{19}$

Otro ejemplo similar en contenido se encuentra en la columna de opinión de Aldo Mariátegui titulada "Supa no supo" (2009). Hilaria Supa, a quien Mariátegui menciona muy creativamente en el título, es una excongresista indígena que representó a la región de Cusco. En el 2009, ella fue fotografiada mientras tomaba apuntes durante una sesión del pleno. Dichos apuntes presentaban errores gramaticales y sintácticos del castellano, y varios medios de comunicación no dudaron en hacer escarnio de dichas faltas sin considerar, por lo menos, que la lengua materna de la excongresista es el quechua. Mariátegui no fue la excepción y la criticó por tener "un nivel cultural tan bajo" y escribir "peor que un niño de ocho años", pero, sobre todo, por "no est[ar] mínimamente iluminad[a] por las luces de la cultura" (Mariátegui, 2009) ${ }^{20}$. Aquí podemos encontrar similitudes con la distinción que hace Mignolo (1995) para la historiografía de finales del siglo XV, a saber, el de una historiografía basada en una teoría de la escritura que excluía otras formas de registrar eventos pasados y otras formas de producir conocimiento.

En ese mismo año, después de los cruentos enfrentamientos en Bagua entre los efectivos de la policía y los comuneros indígenas, el expresidente del Perú, Alan García, se refirió a los últimos como ciudadanos que "no son de primera clase" (citado por Marapi Salas, 2009, 0:22-0:24) y agregó que la postura de estos, en contra de que el Estado autorizara a compañías extranjeras para que perforen sus territorios en busca de petróleo y gas, y a favor de las relaciones ancestrales de dichas comunidades con la naturaleza, nos llevaría "a la irracionalidad y al retroceso primitivo" (citado por Marapi Salas, 2009, 0:34-0:40 $)^{21}$. Comentario que nos lleva a pensar sobre la temporalización evolucionaria que menciona Fabian (2014) y la colonialidad de la naturaleza de Walsh (2007).

19 "The price they must pay for integration is high: renunciation of their culture, their language, their beliefs, their traditions and customs, and the adoption of the culture of their ancient masters. [...] If forced to choose between the preservation of Indian cultures and their complete assimilation, with great sadness I would choose modernization of the Indian population, because there are priorities; and the first priority is, of course, to fight hunger and misery" (Vargas Llosa, 1990, pp. 51-52).

20 Ya sea desde la lingüística o del análisis crítico del discurso (ACD) se analizan las diversas formas con que el racismo se manifiesta en la prensa peruana (Arrunátegui, 2010a; Mamani, 2012). A ello se le puede sumar el sucinto trabajo de Zavala (2007) sobre el racismo en el Perú contemporáneo, en el cual concluye que "[...] es importante considerar que en el Perú este racismo [de tipo diferencialista] coexiste con una jerarquización de tipo colonial que no solo supone una marginación, sino también una explotación desde lo económico y con una realidad de inequidad con fuertes barreras para que todos accedan por igual a las mismas oportunidades sociales" (p. 365).

21 Un análisis discursivo de la representación periodística de los actores involucrados en este conflicto es realizado por Arrunátegui (2010b) y Córdova (2016). A este respecto, Arrunátegui (2010b) expresa: "Los resultados muestran que la prensa peruana utiliza una serie de estrategias discursivas que forman parte de una metaestrategia denominada el cuadrado ideológico (Van Dijk, 1999, 2010), característica de todo discurso ideológico, que tiene por objetivo enfatizar lo positivo del Nosotros (la prensa, el Gobierno y el ciudadano de costumbres occidentales) y enfatizar lo negativo del Otro (los pueblos amazónicos)" (pp. 353-354). 
Si bien los ejemplos mencionados son parte de un discurso moderno que está normalizado y que poseen una gran carga simbólica por provenir de relevantes personajes públicos, nos parece que este tipo de narrativa se puede apreciar de mejor manera a través de los discursos utilizados por la publicidad con base en los estereotipos relacionados con la belleza física (Portocarrero, 2013). Para Bruce (2007), en la misma línea que Quijano (2000), respecto a la noción de raza como uno de los ejes organizadores de la sociedad peruana posconquista, la belleza física en el Perú "ha estado signada por el predominio estricto de patrones eurocéntricos" (Quijano, 2000, p. 68). Y, en la actualidad:

[...] los medios masivos, en particular los publicitarios, desempeñan un papel de enorme incidencia en la propagación de una ideología racista íntimamente vinculada a la apariencia física. Al punto que los cánones estéticos dominantes aparecen como un ingrediente esencial para perpetuar la discriminación racista. (Quijano, 2000, p. 68)

Como se puede apreciar, el problema aquí expuesto no es un problema aislado y circunscrito a nuestra época, sino que data de finales del siglo $\mathrm{XV}$, como destacan Quijano y Walsh, pero también Mignolo. Sin embargo, la gran diferencia radica en que los canales usados para propagar estas diferencias y jerarquías según el concepto de raza (o belleza física) tienen una mayor penetración en la sociedad ${ }^{22}$. Este hecho, por su carácter subrepticio y no coercitivo, a nuestro parecer, cimenta y naturaliza dichas diferencias y jerarquías.

Desde el psicoanálisis, Bruce (2007), a este respecto, expone cómo funciona este tipo de discurso a través del siguiente caso hipotético:

¿Qué puede sentir una joven pobladora de Lima Norte cuando confronta su imagen con la de la modelo Valeria Mazza, representante de los almacenes Saga Falabella en el Perú? Una insalvable diferencia, para comenzar. Pero puede que esto se traduzca también en una sensación de inferioridad, porque lo que se está proponiendo no es solo la imagen de una mujer bella. Lo que dicha imagen comunica es la representación de belleza. Ahí es donde anida el problema. (p. 73)

22 Sobre la penetración de internet y las redes sociales en la región, Elizalde (2019) menciona que, a pesar de las dificultades que muchas personas tienen para satisfacer sus necesidades básicas, "algunos países de la región igualan e incluso superan el uso de redes sociales de países desarrollados, mientras la brecha entre usuarios de internet en América Latina y usuarios de redes sociales es la más estrecha del mundo - la penetración de internet es del $68 \%$, mientras que la penetración de los medios sociales es del $63 \%$, apenas un $5 \%$ de diferencia (Statista, 2018)-. De los diez países con mayor tiempo utilizado en redes sociales, cinco de ellos fueron latinoamericanos, ranking que fue liderado por usuarios brasileños, argentinos y mexicanos con 4 horas al día (CEPAL, 2018b)" (p. 105). 


\section{COMUNICACIÓN DECOLONIZADORA}

Si los medios de comunicación, entendidos como vehículos de representaciones sociales, están moldeados por la "producción de un imaginario moderno/colonial/capitalista/ blanco/heterosexual/patriarcal desde el cual se configura y clasifica a la población" (Maldonado, 2013, p. 470), ¿qué implica optar por una postura decolonizadora de la comunicación? Implicaría, antes que nada, una postura ética. Ética porque la decolonización parte de la precariedad, de la exclusión, de la carencia de una vida digna de muchas personas para construir las bases de espacios de diálogo, intercambio y coexistencia solidarios y honestos entre diversos mundos; por ello, se sitúa en oposición a la visión totalizante y homogeneizadora de la modernidad/colonialidad ${ }^{23}$. En ese sentido, una comunicación decolonizadora sería "la opción epistémica y liberadora que se plantea como estrategia de subversión del actual régimen de visualidad configurado para representar la otredad" (Maldonado, 2013, p. 471). Este actual régimen sería lo que Barriendos (2011) denomina la "colonialidad del ver", es decir, "las maquinarias visuales de racialización que han acompañado el desarrollo del capitalismo moderno/colonial" (p. 26), como, por ejemplo, la construcción de la imagen del salvaje en el "Nuevo Mundo".

Sin embargo, hay que considerar que el contexto en el que ahora se sitúa esta colonialidad del ver se enmarca en lo que el filósofo colombiano Santiago Castro-Gómez llama "capitalismo cognitivo". En la línea de trabajos como los de Baudrillard (1984, 2002), Bourdieu (1997) y Sibilia (2013) en torno a la importancia de la imagen en una sociedad donde prima la visibilización, hipermediatización y espectacularización (Muro Ampuero, 2015), el capitalismo cognitivo haría referencia a "una nueva forma de organización del trabajo [donde se] valoriza la dimensión simbólica, social, afectiva, comunicativa y emocional de las formas comunadas, proyectando una nueva ciudadanía cultural" (Sierra, 2016, p. 14). En ese sentido, el control de los medios de representación tendría un rol protagónico en la construcción de las imágenes de las subalternidades.

Así, diversos autores y autoras comparten la idea de que la comunicación, en su funcionamiento actual, ha servido como plataforma de la modernidad, de la colonialidad y del capital para difundir estereotipos en donde se representa al Otro negativamente (Barranquero Carretero y Sáez Baeza, 2015; Barriendos, 2011; Castro Lara, 2016; Maldonado, 2013; Sierra, 2016). Por eso:

Decolonizar la iconosfera requiere de la incorporación de nuevos relatos visuales, desprendidos de las lógicas de producción de los medios de comunicación hegemónicos. Para decolonizar la iconosfera es necesario que los actores sociales y

23 No obstante, ello no significa que los valores éticos de los grupos subalternos ubicados dentro del proyecto decolonizador sean intrínsecamente buenos. Ello se debe tener siempre en cuenta desde una postura crítica y autorreflexiva con el objetivo de no caer en esencialismos. 
culturales se apropien de los diversos dispositivos de producción del imaginario, de modo de instalar formas-otras de representación que amplíen y/o se contrapongan a la iconosfera que actualmente visualiza al "otro" desde el prima [sic] de la colonialidad del ver. [...] No se trata tan solo de erigir imágenes-alternativas, se trata también de generar retóricas visuales que narren la historia reconociendo las heridas dejadas por el paso de la colonialidad sobre los colectivos humanos que han debido sufrir la dominación sobre sus cuerpos y sus representaciones. (Maldonado, 2013, p. 472)

De este fragmento podemos discutir varios puntos en relación con la comunicación decolonizadora. En primer lugar, este tipo de comunicación necesita politizarse. La politización de la comunicación implica que esta tome una postura crítica en torno a la modernidad/colonialidad y a las relaciones de poder que de este binomio emanan; supone, en segundo lugar, que se piensen nuevas lógicas epistémicas y estéticas dentro del campo comunicacional; $y$, en tercer lugar, dar espacio a nuevos saberes (teniendo en cuenta el debate entre saber y conocimiento/ciencia) para que participen de la iconosfera y deslindarse de las lógicas mercantiles que valorizan al mercado sobre lo humano (Barranquero Carretero y Sáez Baeza, 2015, p. 55; Castro Lara, 2016, p. 116; Maldonado, 2013, p. 474).

Estos tres puntos representan un bloque interdependiente que reúne a las cuatro esferas de la colonialidad señaladas por Walsh (colonialidad del poder, del saber, del ser y de la naturaleza) y, además, a la mencionada por Barriendos (la colonialidad del ver) como puntos neurálgicos para poder visibilizar a unos saberes "otros", unas racionalidades "otras", unas estéticas "otras", etcétera, desde diversos locus de enunciación (Castro Lara, 2016).

Resulta así casi imperativo establecer un itinerario decolonial-comunicacional con una fuerte impronta indígena, afroamericana, campesina, feminista, homosexual, como parte de las configuraciones históricas, de los nombres del y en el tiempo, que incitan a la memoria y simbolizan el tiempo-espacio-movimiento (y quién sabe qué otras categorías no occidentales) que ayuden a articular unos otros conocimientos que redefinan los espacios, las relaciones y las formas de enunciación. (p. 115)

Esto se refuerza si entendemos a la comunicación como un intercambio intersubjetivo horizontal y activo entre las partes involucradas en dicho proceso, en donde el reconocimiento del Otro, y, por consiguiente, su inclusión en el acto comunicativo, es condición esencial para que esta se produzca.

Reconocer a un otro $=$ alius como otro $=$ alter es una condición de comunicación e interacción, por lo tanto, de participar en prácticas socioculturales (o cualquier categoría sociológica, de grupo a sociedad, que se aplique), o de compartir un Lebenswelt [mundo de la vida]. Sin alteridad, no hay cultura, no hay Lebenswelt. 
Incluso en el pensamiento fenomenológico, asumo que este concepto tiene sentido solo si Lebenswelt existe en el plural. (Fabian, 2014, pp. 178-179) ${ }^{24}$

De esa manera, una comunicación decolonizadora tendría que ser, necesariamente, polifónica, un pluriverso de voces que interactúan entre sí y que dialogan entre sí (Gómez, 1983, p. 48). Considerando que, como enfatiza Nealon (1997) respecto a la teoría del dialogismo de Bajtín, dialogar no es solo el intercambio de voces en el acto comunicativo en un plano meramente discursivo, sino una postura ética intersubjetiva donde uno (como también el Otro) está en la obligación de escuchar y responder (pp. 131-133). Sin embargo, mientras existan desigualdades en la apropiación, uso y penetración de los diversos medios de representación, este escuchar y responder no podrá ser realizado de una manera honesta, es decir que la polifonía deseada en la comunicación decolonizadora seguirá siendo prácticamente monológica en tanto las relaciones intersubjetivas giren en torno a concepciones modernas/coloniales/capitalistas/heterosexuales/patriarcales ${ }^{25}$.

\section{REFLEXIONES FINALES}

La idea inicial de este trabajo fue mostrar algunos puentes entre las representaciones de los indígenas (pero, en general, de la Otredad) en la forma como se concibió la historia en el "Nuevo Mundo" y las representaciones que podemos apreciar ahora a través de los distintos medios de representación, sobre todo, en los medios de comunicación. De esa manera, podemos decir que el problema de la decolonización de la historia sobre los indígenas (o Indian histories, según Salomon) no es un problema tan alejado del que se presenta en la actualidad en los medios de representación.

Así, se puede concluir que ambos contextos conciben efectos similares a través del uso de los canales predominantes de comunicación de sus épocas. Por un lado, la historia sobre los indios se basó en una aproximación epistémica que venía de "Occidente", en la que predominaba una cierta teoría de la escritura y la literacidad, que excluía a todo saber que no contase con dicha aproximación al conocimiento. Además, de acuerdo con las relaciones de poder ya estructuradas en el "Nuevo Mundo", este paradigma usó los canales que consideraba más importantes para su teoría de la escritura (por ejemplo,

24 "Recognizing an other = alius as other = alter is a condition of communication and interaction, hence of participating in social-cultural practices (or whatever sociological categories, from group to society, apply), or of sharing a Lebenswelt. Without alterity, no culture, no Lebenswelt. Even in phenomenological thought, I assume, this concept makes sense only if Lebenswelt exists in the plural" (Fabian, 2014, pp. 178-179).

25 Con ello no se quiere decir que no existan espacios en donde el Otro pueda expresarse y autorrepresentarse, los hay y lo hacen (por ejemplo, revisar Virtanen, 2015), sino que no existen las condiciones necesarias para que se pueda entablar un diálogo (revisar la segunda nota a pie de página). 
las cartas, las crónicas, las enciclopedias, entre otras) como medios en donde se legitimaban ciertas representaciones (negativas) de lo indígena.

En la actualidad, los medios de representación (con una mayor variedad y, a su vez, mayor penetración en la población) presentan problemas e interrogantes similares. El problema radica en que nuestras sociedades (las latinoamericanas) adolecen de prácticas y discursos coloniales, modernos y capitalistas. Pero el problema se intensifica aún más cuando percibimos que el dominio no solo se manifiesta materialmente (y con mayor violencia en la carne del Otro periférico), sino que también, y siguiendo la propuesta gramsciana de construcción de hegemonía, se revela simbólicamente por medio de discursos hegemónicos diseminados a través de los diversos medios de representación (instituciones, formas de construir conocimiento, formas de estar en el mundo, medios de comunicación, etcétera).

De esa manera, el discurso hegemónico capitalista/moderno/colonial/patriarcal/ heterosexual no solo construye la imagen del Otro desde sus propios ojos homogeneizadores y jerarquizantes, sino que también le niega al Otro el acceso a tener una voz con el mismo alcance en la población, relegándolo a un plano de resistencia a través de medios alternativos (o las redes sociales, en la actualidad). Por eso, una comunicación decolonizadora es imperante como una propuesta teórica democrática, anticapitalista, decolonial (del poder, del saber, del ser, de la naturaleza y del ver), antieurocéntrica y feminista en la que el Otro se constituya (y lo constituyan) en una alteridad positiva y dialógica.

\section{REFERENCIAS}

Alarcón, L., y Torres, K. (2015). Cholas de pantalla: estereotipos de la mujer andina en los programas de humor de la televisión peruana. En VIII Seminario Regional (Cono Sur) ALAIC: "Políticas, actores y prácticas de la comunicación: encrucijadas de la investigación en América Latina". Córdoba: Escuela de Ciencias de la Información.

Arriaga, E. (2013). Racismo y discurso en la era digital: el caso de la revista Hola y los discursos en las redes sociales. Discurso y Sociedad, 7(4), 617-642.

Arrunátegui, C. (2010a). El racismo en la prensa escrita peruana. Un estudio de la representación del Otro amazónico desde el análisis crítico del discurso. Discurso y Sociedad, 4(3), 428-470.

Arrunátegui, C. (2010b). Ideología y prensa escrita en el Perú: el caso Bagua. Lexis, 34(2), 353-368.

Barranquero Carretero, A., y Sáez Baeza, C. (2015). Comunicación y buen vivir. La crítica descolonial y ecológica a la comunicación para el desarrollo y el cambio social. Palabra Clave, 18(1), 41-82. 
Barriendos, J. (2011). La colonialidad del ver. Hacia un nuevo diálogo visual interepistémico. Nómadas, 35, 13-29.

Baudrillard, J. (1984). Las estrategias fatales. Barcelona: Anagrama.

Baudrillard, J. (2002). La ilusión vital. Buenos Aires: Siglo Veintiuno Editores.

Bauer, R. (2001). "EnCountering" Colonial Latin American Indian chronicles. Felipe Guaman Poma de Ayala's history of the "New World". American Indian Quaterly, 25(2), 274-304.

Bourdieu, P. (1997). Sobre la televisión. Barcelona: Anagrama.

Bruce, J. (2007). Nos habíamos choleado tanto. Psicoanálisis y racismo. Lima: Universidad de San Martín de Porres.

Bubnova, T. (1997). El principio ético como fundamento del dialogismo en Mijaíl Bajtín. Escritos, Revista del Centro de Ciencias del Lenguaje, 15-16, 259-273.

Castro Lara, E. (2016). Reflexiones para decolonizar la cultura académica latinoamericana en comunicación. Chasqui, Revista Latinoamericana de Comunicación, 131, 107-122.

CEPAL, CETiC.br y NiC.br (2018). Monitoreo de la Agenda Digital para América Latina y el Caribe eLAC2018. Santiago de Chile: Naciones Unidas.

Córdova, A. (2016). Selbst-und Fremdzuschreibung in ländlichen sozialen Konflikten in Peru und ihre Darstellung in den Medien am Beispiel des sozialen Konfliktes von Bagua (tesis de maestría). Universidad de Bonn, Alemania.

De Castro, J. (2010). Mario Vargas Llosa versus barbarism. Latin American Research Review, 45(2), 5-26.

De Sousa Santos, B. (2012). Cuando los excluidos tienen derecho: justicia indígena, plurinacionalidad e interculturalidad. En B. de Sousa Santos y J. L. Exeni Rodríguez (Eds.), Justicia indígena, plurinacionalidad e interculturalidad en Bolivia (pp. 11-48). Quito: Abya-Yala; Fundación Rosa Luxemburg.

Dettleff, J. (2015). Construcción de estereotipos en la comedia peruana. El caso de La paisana Jacinta. En VIII Seminario Regional (Cono Sur) ALAIC: "Políticas, actores y prácticas de la comunicación: encrucijadas de la investigación en América Latina". Córdoba: Escuela de Ciencias de la Información.

Dussel, E. (2007). Materiales para una política de la liberación. México D. F.: Facultad de Filosofía, UANL; Plaza y Valdés Editores.

Elizalde, R. M. (2019). Colonialismo 2.0 en América Latina y el Caribe: ¿qué hacer? En M. Caciabue y K. Arkonada (Coords.), Más allá de los monstruos. Entre lo viejo que 
no termina de morir y lo nuevo que no termina de nacer (pp. 102-117). Río Cuarto: UniRío Editora.

Fabian, J. (2014). Time \& the other. How anthropology makes its object. Nueva York: Columbia University Press.

Ferrándiz, J., Ibáñez, C., y Espinosa, A. (2011). Racismo 2.0: expresiones de prejuicio en las redes sociales virtuales tras las elecciones generales 2011. Comunicación Política, 2(3), 75-83.

Gómez, F. (1983). El concepto de "dialoguismo" en Bajtín. La otra forma del diálogo renacentista. 1616: Anuario de la Sociedad Española de Literatura General y Comparada, 5(1), 47-54.

González, J., y Arteaga, M. (2005). Monitoreo de medios. La representación de lo indígena en los medios de comunicación. Convenio en minga con los pueblos indígenas y por el derecho a su palabra. Santiago de Cali: Hombre Nuevo Editores.

Iannuzzi, I. (2008). Talavera y Nebrija: lenguaje para convencer, gramática para pensar. HISPANIA. Revista Española de Historia, 68(228), 37-62.

Kokotovic, M. (2000). Vargas Llosa in the Andes: the racial discourse of neoliberalism. Confluencia, 15(2), 156-167.

Lonetree, A. (2012). Decolonizing museums: representing Native America in National and Tribal Museums. Chapel Hill, NC: University of North Carolina Press.

Maldonado, C. (2013). Decolonizar la iconosfera. Medios de comunicación alternativos en la producción de un imaginario contrahegemónico. En T. Dodds y R. Ramírez (Eds.), La imagen en las sociedades mediáticas latinoamericanas. Actas de la IX Bienal Iberoamericana de Comunicación (pp. 470-475). Santiago de Chile: Instituto de la Comunicación en Imagen de la Universidad de Chile; Red Académica Iberoamericana de Comunicación.

Mamani, L. (2012). Actitudes lingüísticas y formación docente. Lengua y Sociedad, 12(1), 153-166.

Manrique Tisnés, H. (2008). Saber y conocimiento: una aproximación plural. Acta Colombiana de Psicología, 11(2), 89-100.

Marapi Salas, R. (2009). Alan García y los ciudadanos de primera clase. Bagua Perú [archivo de video]. Recuperado de https://www.youtube.com/watch?v=3ekPeb6nMnw

Mariátegui, A. (23 de abril del 2009). Supa no supo [columna de opinión]. Diario Correo. Recuperado de https://diariocorreo.pe/opinion/supa-no-supo-142081/. 
Mignolo, W. (1994). Afterword: writing and recorded knowledge in colonial and postcolonial situations. En E. Hill Boone y W. Mignolo (Eds.), Writing without words: alternative literacies in Mesoamerica and the Andes (pp. 293-313). Durham, NC: Duke University Press.

Mignolo, W. (1995). The darker side of the Renaissance: literacy, territoriality, and colonization. Ann Arbor, Ml: University of Michigan Press.

Muro Ampuero, E. (2015). Análisis de las configuraciones y articulaciones del campo político en la "generación\#". Contratexto, 24, 77-93. doi:10.26439/contratexto2015. n024.588

Nealon, J. (1997). The ethics of dialogue: Bakhtin and Levinas. College English, 59(2), $129-148$.

Pérez, M., y Argueta, A. (2011). Saberes indígenas y diálogo intercultural. Revista Mexicana de Sociología, 5(10), 31-55.

Pineda, E. (2016). Discriminación racial y vida cotidiana en América Latina: empleo, educación y medios de comunicación. Revista Venezolana de Análisis de Coyuntura, 22(2), 121-144.

Portocarrero, G. (2013). La utopía del blanqueamiento y la lucha por el mestizaje. En A. Grimson y K. Bidaseca (Coords.), Hegemonía cultural y políticas de la diferencia (pp. 165-200). Buenos Aires: CLACSO.

Quijano, A. (2000). Colonialidad del poder, eurocentrismo y América Latina. En E. Lander (Ed.), La colonialidad del saber: eurocentrismo y ciencias sociales. Perspectivas latinoamericanas (pp. 201-246). Buenos Aires: CLACSO.

Rivera Cusicanqui, S. (2016). Lo verdaderamente indio está dentro de todos nosotros. Suplemento Ojarasca, 235, 4-5.

Said, E. (2016). Orientalismo. México, D. F.: Penguin Random House.

Salomon, F. (1999). Testimonies: the making and reading of Native South American historical sources. En F. Salomon y S. B. Schwartz (Eds.), Cambridge history of the native peoples of the Americas (vol. III, South America, part. 1, pp. 19-95). Cambridge: Cambridge University Press.

Santos Granero, F. (1998). Writing history into the landscape: space, myth, and ritual in contemporary Amazonia. American Ethnologist, 25(2), 128-148.

Santos-Granero, F. (2004). Arawakan sacred landscapes. Emplaced myths, place rituals, and the production of locality in Western Amazonia. En E. Halbmayer y E. Mader (Eds.), Kultur, Raum, Landschaft. Zur Bedeutung des Raumes in Zeiten der Globalität (pp. 93-122). Fráncfort del Meno: Brandes \& Apsel Verlag. 
Sibilia, P. (2013). La intimidad como espectáculo. Buenos Aires: Fondo de Cultura Económica.

Sierra, F. (2016). Comunicación y buen vivir. Nuevas matrices teóricas del pensamiento latinoamericano. Chasqui, Revista Latinoamericana de Comunicación, 131, 9-18.

Tuhiwai, L. (2008). Decolonizing methodologies: research and indigenous peoples. Nueva York: Zed Books.

United States Senate Committee on Foreign Relations. (2011). Latin American governments need to "friend" social media and technology. Recuperado de https://www.gpo. gov/fdsys/pkg/CPRT-112SPRT70501/html/CPRT-112SPRT70501.htm

Vargas Llosa, M. (1990). Questions of Conquest. Harpers, December, 45-53.

Virtanen, P. (2015). Indigenous social media practices in Southwestern Amazonia. Alter Native, 11(4), 350-362.

Walsh, C. (2007). ¿Son posibles unas ciencias sociales/culturales otras? Reflexiones en torno a las epistemologías decoloniales. Nómadas, 26, 102-113.

Walsh, C. (2009). Interculturalidad, Estado, sociedad. Luchas (de)coloniales de nuestra época. Quito: Universidad Andina Simón Bolívar; Abya-Yala.

Zavala, V. (2007). Yo te segrego a ti porque tu falta de educación me ofende: una aproximación al discurso racista en el Perú contemporáneo. En T. Van Dijk (Ed.), Discurso y racismo en América Latina (pp. 333-369). Barcelona: Gedisa. 